\title{
Polymer density functional approach to efficient evaluation of path integrals
}

\author{
Brukhno, Andrey; Vorontsov-Velyaminov, Pavel N.; Bohr, Henrik
}

Published in:

Physical Review E

Link to article, DOI:

10.1103/PhysRevE.72.046703

Publication date:

2005

Document Version

Publisher's PDF, also known as Version of record

Link back to DTU Orbit

Citation (APA):

Brukhno, A., Vorontsov-Velyaminov, P. N., \& Bohr, H. (2005). Polymer density functional approach to efficient evaluation of path integrals. Physical Review E, 72(4), 046703. https://doi.org/10.1103/PhysRevE.72.046703

\section{General rights}

Copyright and moral rights for the publications made accessible in the public portal are retained by the authors and/or other copyright owners and it is a condition of accessing publications that users recognise and abide by the legal requirements associated with these rights.

- Users may download and print one copy of any publication from the public portal for the purpose of private study or research.

- You may not further distribute the material or use it for any profit-making activity or commercial gain

- You may freely distribute the URL identifying the publication in the public portal

If you believe that this document breaches copyright please contact us providing details, and we will remove access to the work immediately and investigate your claim. 


\title{
Polymer density functional approach to efficient evaluation of path integrals
}

\author{
Andrei Broukhno, ${ }^{1,2}$ Pavel N. Vorontsov-Velyaminov, ${ }^{2}$ and Henrik Bohr ${ }^{1}$ \\ ${ }^{1}$ Quantum Protein Centre, Technical University of Denmark, Building 309, DK-2800 Lyngby, Denmark \\ ${ }^{2}$ Molecular Biophysics, Faculty of Physics, SPbSU, 198504 Unlyanovskaya-1, Petrodvoretz, Russia
}

(Received 12 May 2005; published 3 October 2005)

\begin{abstract}
A polymer density functional theory (P-DFT) has been extended to the case of quantum statistics within the framework of Feynman path integrals. We start with the exact P-DFT formalism for an ideal open chain and adapt its efficient numerical solution to the case of a ring. We show that, similarly, the path integral problem can, in principle, be solved exactly by making use of the two-particle pair correlation function (2p-PCF) for the ends of an open polymer, half of the original. This way the exact data for one-dimensional quantum harmonic oscillator are reproduced in a wide range of temperatures. The exact solution is not, though, reachable in three dimensions (3D) because of a vast amount of storage required for 2p-PCF. In order to treat closed paths in 3D, we introduce a so-called "open ring" approximation which proves to be rather accurate in the limit of long chains. We also employ a simple self-consistent iteration so as to correctly account for the interparticle interactions. The algorithm is speeded up by taking convolutions with the aid of fast Fourier transforms. We apply this approximate path integral DFT (PI-DFT) method to systems within spherical symmetry: 3D harmonic oscillator, atoms of hydrogen and helium, and ions of $\mathrm{He}$ and $\mathrm{Li}$. Our results compare rather well to the known data, while the computational effort (some seconds or minutes) is about 100 times less than that with Monte Carlo simulations. Moreover, the well-known "sign problem" is expected to be considerably reduced within the reported PI-DFT, since it allows for a direct estimate of the corresponding partition functions.
\end{abstract}

DOI: 10.1103/PhysRevE.72.046703 PACS number(s): 02.70. $-\mathrm{c}$, 31.15.Kb, 31.15.Ew, 05.30. $-\mathrm{d}$

\section{INTRODUCTION}

The density functional approach has proven to be a very useful tool in investigation of both quantum and classical systems. Initially, the variational principle for the ground state energy of inhomogeneous electron gas was derived in the pioneering works by Kohn, Hohenberg, and Sham $[1,2]$ which was soon generalized to the case of nonzero temperature by Mermin [3]. The same ideas led to development of the density functional theory (DFT) for nonuniform atomic fluids by Lebowitz and Percus [4], and a similar methodology was later applied to polymer solutions by the group of Chandler $[5,6,8]$. Starting with these igniting works, a fistful of DFT's appropriately treating polymers have been further developed and successfully applied, mostly in the area of surface phenomena [12-16,18-21].

While the quantum density functional theory has become nowadays the primary instrument for quantum chemical calculations, its numerical algorithms are known to suffer from a rather unfavorable scaling with the number of particles in the system, $O\left(N^{3}\right)$ at best. Apart from that, the exact form of the kinetic contribution to the electron density functional, in general, is not available, except for the case of freely moving particles. Thus, the "excess" kinetic part is usually combined with an effective exchange-correlation term to be separately investigated in each particular case. Obviously, this constitutes an additional source of systematic errors and requires some extra effort to minimize those.

On the other hand, numerical methods of classical DFT's are known to be very efficient and accurate and, hence, might be utilized for path integral (PI) evaluation within Feynman's formulation of quantum statistics [22,23]. In fact, this possi- bility is often discussed in works on polymer DFT's $[8,19]$. Moreover, already in the late 1980's a successful application of the PI isomorphism within the classical DFT framework was reported by McCoy et al. [9-11], who developed a path integral DFT of freezing for quantum liquids. Earlier, the quantum dispersion effect was incorporated within the reference interaction site model (known as RISM) by Chandler et al. [7], with an application to the primitive hard sphere ring model of helium fluid. Recently, Chong Gu et al. [17] have applied the fundamental measure theory (known as FMT), proposed earlier by $\mathrm{Yu}$ and $\mathrm{Wu}$ [16], to study adsorption of hydrogen molecules into nanopores. In such studies, however, the quantum fluid density is usually approximated up to the second order about the reference uniform system, while the focus is on the uncertainties in particle positions. Thus, a general path integral DFT that should be applicable to electronic structure on the atomic scale, with account for the exchange interaction, is not yet available.

Nevertheless, the evident success of the aforementioned methods encouraged us to address the general quantum statistical problem from the standpoint of a classical polymer DFT. In this work we present a path integral DFT (PI-DFT) approach that is not limited to the second order approximation. It also reduces computer demand by orders of magnitude, while providing a striking precision, at least as compared to the corresonding Monte Carlo simulations [24-27].

The main idea behind most of the classical DFT methods is to reduce a many particle problem to a single particle one [18]. Being based on the existence of a one-particle grand canonical free energy functional, this procedure proved to be plausible for systems where local density does not deviate too much from the uniform distribution. The goal is usually accomplished by introducing an appropriate effective (ex- 
cess) free energy functional per site in the fluid, which makes it possible to find the equilibrium one particle distribution in the course of a self-consistent iteration that minimizes the free energy. It is common to derive such functionals (the excess part thereof) from the equation of state for the corresponding bulk solution, and that is why these approaches can be considered as being partly phenomenological, which inevitably brings certain hesitations. However, as Woodward first pointed out [12], such a DFT is not necessarily to be approximate, nor the bonding interaction within a polymer is necessarily to be included within the effective excess contribution. That is, since bonds only involve the nearest neighbor pairs along the chain, it is possible to explicitly integrate over the monomer positions by use of a subsequent (recurrent) integration scheme [16]. Thus, unlike with other polymer DFT's [33], within Woodward's framework the problem of ideal chain in external field is solved exactly, for the expense of some extra computer time though.

As argued in the literature [19], Woodward's technique cannot be applied directly to the path integral problem, because the recurrent procedure mentioned above is only suited for linear chains with free ends, but not for closed, or ring, polymers which the path integral formalism is based on. The shortcoming is not, though, too crucial since in the limit of infinite chain length each half of the ring obeys the same statistics as an independent chain with loose ends [28]. This observation leads us to a very useful approximation that we employ in order to evaluate path integrals, while still being able to make use of the explicit recurrent integration of the bonding potential. Besides, where possible we speed up the numerical algorithm by solving the arising convolution integrals in Fourier space, with the aid of fast Fourier transforms.

Furthermore, we avoid introducing any effective excess free energy term due to inter-particle interactions, which is, anyhow, very unlikely to work in general case because of the intrinsic nonuniformity of the problem. Instead, we organize a self-consistent iteration as to account for the interaction between vertices with the same index on trajectories corresponding to different particles. Our calculations in the case of two distinguishable particles (in electron subsystems of $\mathrm{He}$ atom and $\mathrm{Li}^{+}$ion) show that the convergence is reached already after a few $(<6)$ iterations and the obtained particle distributions contain errors within $2 \%$, at worse.

The remainder of the paper is organized as follows. In Sec. II we start with a brief outline of the Feynman path integral formulation, and in the subsequent Secs. II B-II G we provide the foundations as well as testimonial for our approach. The computational details and discussion of the results obtained for a number of atomic systems can be found in Sec. III. Section IV finalizes the paper with our conclusions and discussion on possible future applications of the suggested PI-DFT.

\section{THEORY AND METHOD}

There is substantial literature on the path integral formulation of quantum statistics as well as on the numerical methods that have been utilized within this framework, mostly Fourier and discretized PI Monte Carlo as well as the Bead-
Fourier combination [24-27,29-31]. Therefore, below we only recapitulate the essential formulas and move on straight to the suggested PI-DFT method.

\section{A. Discretized path integral representation}

As is well known, Feynman's approach establishes an isomorphism between the quantum statistical (QS) canonical problem of a quantum particle in an external field and the classical statistical problem of a closed polymer chain in the same field. The quantum partition function of a particle is determined by its density matrix in coordinate presentation,

$$
Z(\beta) \equiv \operatorname{Tr} \hat{\rho}(\beta)=\sum_{i} e^{-\beta E_{i}}=\int \rho(\mathbf{r}, \mathbf{r} ; \beta) d \mathbf{r},
$$

where

$$
\rho\left(\mathbf{r}, \mathbf{r}^{\prime} ; \beta\right) \equiv \sum_{i} \psi_{i}^{*}\left(\mathbf{r}^{\prime}\right) e^{-\beta E_{i}} \psi_{i}(\mathbf{r})=\left\langle\mathbf{r}^{\prime}\left|e^{-\beta \hat{H}}\right| \mathbf{r}\right\rangle .
$$

The identity, $e^{-\beta \hat{H}}=\left(e^{-(\beta / n) \hat{H}}\right)^{n}$, allows one to formally treat the system at an arbitrarily high effective temperature, $T_{n}$ $=n T$, or low $\beta_{n}=1 /\left(n k_{B} T\right)$ ( $k_{B}$ is Boltzmann constant), by reexpressing the density matrix diagonal elements as

$$
\rho\left(\mathbf{r}_{0}, \mathbf{r}_{n}=\mathbf{r}_{0} ; \beta\right)=\int \rho\left(\mathbf{r}_{0}, \mathbf{r}_{1} ; \beta_{n}\right) \ldots \rho\left(\mathbf{r}_{n-1}, \mathbf{r}_{n} ; \beta_{n}\right) d \mathbf{r}_{1} \ldots d \mathbf{r}_{n-1} .
$$

Then, due to Trotter relation,

$$
e^{-(\beta / n) \hat{H}} \rightarrow e^{-(\beta / n) \hat{T}} e^{-(\beta / n) \hat{V}}, \quad n \rightarrow \infty,
$$

at sufficiently low $\beta_{n}$ each high temperature term in Eq. (2), can be well approximated by

$$
\begin{aligned}
\rho\left(\mathbf{r}_{i}, \mathbf{r}_{i+1} ; \beta_{n}\right) \approx & \left(\frac{n}{\lambda^{2}}\right)^{3 / 2} \exp \left(-\frac{\pi n}{\lambda^{2}}\left(\mathbf{r}_{i+1}-\mathbf{r}_{i}\right)^{2}\right) \\
& \times \exp \left(-\frac{\beta}{n} \phi\left(\mathbf{r}_{i}\right)\right),
\end{aligned}
$$

where the first exponent is the density matrix of a free particle in coordinate presentation at inverse temperature $\beta_{n}$ $=\beta / n, \lambda \equiv \sqrt{\beta h^{2} / 2 \pi \mu}$ is the thermal wave length of the particle, $\mu$ is its mass, and $h$ is the Planck constant.

Thus, a QS state of a particle at $T=$ const is defined by its equilibrium path set that can be considered as a Gaussian ring polymer being in thermal equilibium at $T_{n}=n T$, with its spring rigidity dependent on temperature. Therefore, the approximation is also often called a discretized (or bead) path integral. Clearly, any observable in coordinate presentation is obtained as

$$
\langle O\rangle=Z^{-1} \int\langle\mathbf{r}|\rho \hat{O}| \mathbf{r}\rangle d \mathbf{r}=Z^{-1} \int \rho\left(\mathbf{r}, \mathbf{r}^{\prime} ; \beta\right)\left\langle\mathbf{r}^{\prime}|\hat{O}| \mathbf{r}\right\rangle d \mathbf{r} d \mathbf{r}^{\prime} .
$$

The partition function of $N$ identical quantum particles [34] (i.e., bosons or fermions), $Z_{N}^{(A, S)}$, can be written as a (symmetrical or antisymmetrical) sum of the partition functions of distinguishable particles, $Z_{N}^{(D)}$, over all $N$ ! permutations of the second argument of the density matrix, 


$$
\begin{aligned}
Z_{N}^{(A, S)}(\beta) & =\frac{1}{N !} \sum_{P} \xi^{[P]} Z_{N}^{(D)}(\beta ; P) \\
& =\frac{1}{N !} \sum_{G} \xi^{[G]} K(G) L(G) Z_{N}^{(C)}(\beta ; G) .
\end{aligned}
$$

Here sign factor $\xi= \pm 1$ defines the permutation symmetry (for bosons/fermions), and

$$
Z_{N}^{(D)}(\beta ; P)=\int \rho_{N}\left(\mathbf{R}_{0}, \mathbf{R}_{n}\right)_{\mathbf{R}_{n}=P\left(\mathbf{R}_{0}\right)} d \mathbf{R}_{0},
$$

with $\mathbf{R}_{0}=\left(\mathbf{r}_{0}^{(1)}, \mathbf{r}_{0}^{(2)}, \ldots, \mathbf{r}_{0}^{(N)}\right)$ being the $3 N$-dimensional vector that determines the positions of initial vertices of $N$ trajectories, and $\mathbf{R}_{n}=\left(\mathbf{r}_{n}^{(1)}, \mathbf{r}_{n}^{(2)}, \ldots, \mathbf{r}_{n}^{(N)}\right)$ containing the coordinates of their ends. For the identical permutation each trajectory is closed onto itself, whereas for an arbitrary permutation $P$ a trajectory can be closed onto the initial vertex of another particle. On the RHS of Eq. (4) we switched to the sum over classes of permutations $[25,26]$ where $L(G)$ is the number of equivalent permutations in class $G$, and spin permutation factor $K(G)=2^{\Sigma C_{\nu}(G)}$, with $C_{\nu}(G)$ being the number of cycles of length $\nu$ in class $G$.

In general, the potential energy includes not only interaction with the external field(s) [as in Eq. (3)] but also the interparticle potential. It is worth noting that the latter acts only between "beads" with the same index on every chain (i.e., in the same "virtual time slice" if one treats $\beta_{n}$ as "virtual time"), the fact that considerably simplifies numerical calculations, as compared to actual classical polymer problems.

\section{B. Density functional theory for an ideal polymer}

As any density functional theory (DFT), the polymer DFT starts with an appropriate free energy functional of the density in the system. From the perspective of evaluating path integrals we are, particularly, interested in the (classical) free energy of the ideal ring polymer. To start with, for the ideal (phantom) chain with free ends in external field, the exact free energy functional is available [12],

$$
\begin{aligned}
\beta F^{(i d)}\left[\rho_{n}(\mathbf{R})\right]= & \int \rho_{n}(\mathbf{R})\left(\ln \left[\rho_{n}(\mathbf{R})\right]-1\right) d \mathbf{R} \\
& +\beta \int \rho_{n}(\mathbf{R}) V_{b}(\mathbf{R}) d \mathbf{R} \\
& +\beta \int \rho_{n}(\mathbf{R}) \Phi_{\mathrm{ext}}(\mathbf{R}) d \mathbf{R},
\end{aligned}
$$

where multivector $\mathbf{R}=\left(\mathbf{r}_{0}, \ldots, \mathbf{r}_{n}\right)$ describes the chain configuration, and $\rho_{n}(\mathbf{R})$ is the total density distribution depending on the positions of all the monomers. The first term in Eq. (5) is the ideal entropy contribution, while the second and third ones are due to the intramolecular (harmonic, in our case) bonding potential and the interaction with external field(s) correspondingly,

$$
V_{b}(\mathbf{R})=K_{f} \sum_{i=0}^{n-1}\left(\mathbf{r}_{i+1}-\mathbf{r}_{i}\right)^{2},
$$

$$
\Phi_{\text {ext }}(\mathbf{R})=\sum_{i=1}^{n} \phi\left(\mathbf{r}_{i}\right)
$$

where, in the PI case, $\beta$ should be replaced by $\beta_{n}=\beta / n$ and $K_{f}=\pi n^{2} / \beta \lambda^{2}$ [cf. Eq. (3)]. Provided the appropriate normalization constraint for $\rho_{n}(\mathbf{R})$, the free energy functional, Eq. (5), is readily minimized to give the equilibrium monomer density depending on the position of any monomer (on average) [12-16],

$$
\begin{aligned}
\rho(\mathbf{r})= & \int \sum_{i=1}^{n} \delta\left(\mathbf{r}-\mathbf{r}_{i}\right) \rho_{n}(\mathbf{R}) d \mathbf{R} \\
\sim & \int \sum_{i=1}^{n} \delta\left(\mathbf{r}-\mathbf{r}_{i}\right) \exp \left[-\beta V_{b}(\mathbf{R})\right] \\
& \times \exp \left[-\beta \Phi_{\text {ext }}(\mathbf{R})\right] d \mathbf{R},
\end{aligned}
$$

where the normalization factor is determined either by the polymer chemical potential in bulk or by the number of chains, when the bulk density vanishes, like in our case.

Formally, Eq. (6) is the exact solution and, when known, it allows one to calculate any property of the system in coordinate presentation. Thus, the major problem in polymer DFT is to find a computationally convenient route to $\rho(\mathbf{r})$. In the case of ideal chain it is possible to rewrite Eq. (6) in a way suitable for practical calculations. That is, due to the fact that the intrachain interactions only comprise the nearestneighbor pairs, it is possible to decouple the integral into the following recurrence [16],

$$
G^{(0)}(\mathbf{r})=1,
$$

$$
\begin{aligned}
G^{(i)}(\mathbf{r})= & \int G^{(i-1)}\left(\mathbf{r}^{\prime}\right) \exp \left[-\beta \phi\left(\mathbf{r}^{\prime}\right)\right] \\
& \times \exp \left[-\beta K_{f}\left(\mathbf{r}-\mathbf{r}^{\prime}\right)^{2}\right] d \mathbf{r}^{\prime}, i>0 .
\end{aligned}
$$

It is clear that, upon $n$ iterations, $p^{(n)}(\mathbf{r})$ $\sim \exp [-\beta \phi(\mathbf{r})] G^{(n)}(\mathbf{r})$ gives the probability density of finding one of the chain ends in position $\mathbf{r}$. The corresponding probability density for $i$-th intermediate monomer, $1<i<n$, is then $p^{(i)}(\mathbf{r}) \sim \exp [-\beta \phi(\mathbf{r})] G^{(i)}(\mathbf{r}) G^{(n-i)}(\mathbf{r})$, where the internal symmetry of the chain about its middle is presumed. Finally, the total monomer distribution, Eq. (6), is reexpressed as

$$
\rho_{\text {open }}^{\text {(exact) }}(\mathbf{r}) \sim \sum_{i=1}^{n} \exp [-\beta \phi(\mathbf{r})] G^{(i)}(\mathbf{r}) G^{(n-i)}(\mathbf{r}) .
$$

Equations (7) and (8) provide an efficient recipe for the exact solution of the problem. In general, the iteration involves only three-dimensional integrals taken one after another. The amount of calculations is then proportional to $3 n$, while the straightforward integration would require of the order of $3^{n}$ operations, which is usually the reason for using Monte Carlo methods. Moreover, with the aid of fast Fourier transforms (FFT), evaluation of the convolution integrals in Eq. (7) can be further speeded up, although a smart account for the spacial symmetry of the system is always worthwhile. 


\section{Exact solution for a ring}

The analogous problem for a closed chain can, in principle, be solved exactly in the following manner. First, one should notice that if the two particle correlation function for the ends of half of the ring, $g_{n / 2}\left(\mathbf{r}, \mathbf{r}^{\prime}\right)$, is known, then the monomer density is obtained simply as

$$
\rho_{\text {ring }}^{\text {(exact) }}(\mathbf{r}) \sim \exp [-\beta \phi(\mathbf{r})] \int \exp \left[-\beta \phi\left(\mathbf{r}^{\prime}\right)\right]\left[g_{n / 2}\left(\mathbf{r}, \mathbf{r}^{\prime}\right)\right]^{2} d \mathbf{r}^{\prime} .
$$

In order to calculate $g_{n / 2}\left(\mathbf{r}, \mathbf{r}^{\prime}\right)$ one can use an iterative procedure similar to that described above. Namely,

$$
\begin{gathered}
g_{1}^{(0)}\left(\mathbf{r}, \mathbf{r}^{\prime}\right) \equiv \exp \left[-\beta K_{f}\left(\mathbf{r}-\mathbf{r}^{\prime}\right)^{2}\right], \\
g_{2}^{(1)}\left(\mathbf{r}, \mathbf{r}^{\prime}\right)=\int \exp \left[-\beta \phi\left(\mathbf{r}^{\prime \prime}\right)\right] g_{1}^{(0)}\left(\mathbf{r}, \mathbf{r}^{\prime \prime}\right) g_{1}^{(0)}\left(\mathbf{r}^{\prime}, \mathbf{r}^{\prime \prime}\right) d \mathbf{r}^{\prime \prime}, \\
g_{4}^{(2)}\left(\mathbf{r}, \mathbf{r}^{\prime}\right)=\int \exp \left[-\beta \phi\left(\mathbf{r}^{\prime \prime}\right)\right] g_{2}^{(1)}\left(\mathbf{r}, \mathbf{r}^{\prime \prime}\right) g_{2}^{(1)}\left(\mathbf{r}^{\prime}, \mathbf{r}^{\prime \prime}\right) d \mathbf{r}^{\prime \prime}, \\
\ldots \\
g_{n / 2}^{(p)}\left(\mathbf{r}, \mathbf{r}^{\prime}\right)=\int \exp \left[-\beta \phi\left(\mathbf{r}^{\prime \prime}\right)\right] g_{n / 4}^{(p-1)}\left(\mathbf{r}, \mathbf{r}^{\prime \prime}\right) g_{n / 4}^{(p-1)}\left(\mathbf{r}^{\prime}, \mathbf{r}^{\prime \prime}\right) d \mathbf{r}^{\prime \prime},
\end{gathered}
$$

where the superscript denotes the iteration number, and the subscript is the number of bonds accounted for on a particular iteration. As is understood from the final formula, after $p$ iterations the correlation function for the ends of the chain with $n / 2=2^{p}$ bonds is obtained. Then, the calculation for the ring polymer consisting of $n=2^{p+1}$ bonds/monomers is accomplished by integrating over the locations of its "middle" monomer, Eq. (9). Obviously, the integral of $\rho_{\text {ring }}^{\text {(exact) }}(\mathbf{r})$ itself gives the corresponding partition function, Eq. (1).

The main disadvantage of this prescription is that it is essentially based on the three-body integrals. Unfortunately, the general 3-dimentional (3D) three-body problem is yet unsolvable with modern computers, mostly due to a huge amount of memory (RAM) needed to store the values of $g\left(\mathbf{r}, \mathbf{r}^{\prime}\right)$. Even under the most favorable assumption of only 100 bins on the grid of each coordinate, the numerical integration in $3 \mathrm{D}$ would require $10^{12}$ floating-point RAM cells, i.e., of the order of thousand gigabites ( $1 \mathrm{~Gb} \sim 10^{9}$ bytes). Nevertheless, we have tested this approach in the case of one-dimensional quantum harmonic oscillator, and our numerical results deviate from the exact ones obtained analytically $[26,27]$ only in the sixth valuable digit, see Table I.

\section{Exact calculations for $1 D$ quantum harmonic oscillator}

The numerically exact solution for monomer density of a ring, Eq. (10), is straightforward to apply. In the case of 1D external field, the problem reduces to evaluation of onedimensional integrals over $r^{\prime \prime}$ for each pair $\left(r, r^{\prime}\right)$ within the interval $\left(-R_{\text {cut }},+R_{\text {cut }}\right)$, where $R_{\text {cut }}$ is chosen as to cover the most important range of non-vanishing probabilities. Fourier transforms are, unfortunately, not applicable in this case.

It should be noted that in order to avoid floating-point overflows and maintain the numerical precision, one has to appropriately scale $g^{(p)}\left(r, r^{\prime}\right)$. We found it safe and convenient to merely normalize $\exp \left[-\beta_{n} K_{f}\left(r-r^{\prime}\right)^{2}\right]$ by the factor of $\left(K_{f} \beta / n \pi\right)^{1 / 2}=\left(n / \lambda^{2}\right)^{1 / 2}$. Thus, its square is used for scaling of $g_{2}^{(1)}\left(r, r^{\prime}\right)$, so that finally the power of $n / 2$ is accumulated, which brings the right norm-factor for the $1 \mathrm{D}$ partition function.

The results for $1 \mathrm{D}$ quantum harmonic oscillator obtained in the range of $\beta^{*}=\beta \hbar \omega=1 / 4, \ldots, 8$ (in intrinsic HO units) are presented in Table I. One should remember that with increasing $n$ the width of the effective harmonic bond is decreasing. Therefore it is wise to cut the numerical integration not only with respect to the vanishing probability in the external field, i.e., where $|r|>R_{\text {cut }}$, but also where the probability of stretched chain diminishes. It is clear that this kind of integration cutoff is meaningful during only few iterations, Eq. (10), until the collective width of $g\left(r, r^{\prime}\right)$ becomes comparable to $R_{\text {cut }}$, and such optimization becomes less efficient with shorter chains and at lower $T$. On the other hand, with longer chains, $n>1000$, one has to use a finer grid in order not to lose the precision. These observations, being illustrated in Fig. 1, explain the nontrivial time scaling in Table I.

\section{Open ring approximation}

Another approach to the problem of ring polymers is to develop a fast approximate method that should converge to the known exact solutions. Since our final aim is the path integral evaluation in the presence of such a strong field as Coulombic one, the convergence with the chain length, $n \rightarrow \infty$, might be quite satisfactory. Below we describe how one can proceed this way.

By looking closely at the sum in Eq. (8), one notices that within an open homogeneous chain the probabilities of finding different monomers in position $\mathbf{r}$ are not equal. Having in mind that in a ring homopolymer all the monomers behave identically, it should be reasonable in this case to equate all the terms in Eq. (8) to that for the innermost monomer,

$$
\rho_{\text {ring }}^{\text {(approx }}(\mathbf{r}) \sim n \exp \left[-\beta_{n} \phi(\mathbf{r})\right]\left[G^{(n / 2)}(\mathbf{r})\right]^{2} .
$$

This appears, in fact, to be a very good approximation for the monomer distribution of the ring chain, and the required convergence is achieved with $n>1000$, as is evident from our results both for the $3 \mathrm{D}$ quantum harmonic oscillator and in the case of simple atomic systems.

It is easy to understand that integration of Eq. (11) gives the partition function (p.f.) for the corresponding open chain, but not that of the ring. However, it can be shown that in the limit of large $n$ the latter approaches the p.f. for two independent open chains twice as short, i.e., separate halves of the ring, $Z_{\text {ring }}(n) \rightarrow Z_{\text {open }}^{2}(n / 2)$ as $n \rightarrow \infty$, e.g., see [28]. This provides us with an appropriate approximation for the p.f. of the ring, in the same convergence regime as with Eq. (11). Besides, in practice one can calculate it in two different ways, without extra CPU demand: $Z_{\text {open }}(n / 2)$ can be ob- 
TABLE I. Results of the numerically exact DFT for the quantum harmonic oscillator, obtained in 1D and presented as to compare with $3 \mathrm{D}$ case. The star symbol, $(\ldots)^{*}$, marks the rows obtained analytically: the cyclic matrix approach [27] was employed for $Z_{1 D}(\beta ; 8)$ and $U_{1 D}^{\text {pot }}(\beta ; 8)$, while $Z_{1 D}(\beta ; \infty)=[2 \sinh (\beta / 2)]^{-1}$ and $U_{1 D}^{\text {pot }}(\beta ; \infty)=\operatorname{coth}(\beta / 2) / 4$, see $[26,27]$. $R_{\text {cut }}$ defines the interval of numerical integration, $\left(-R_{\text {cut }},+R_{\text {cut }}\right)$. The grid number, $N_{\text {grid }}$, was chosen the least still giving the converged values. The last digits of all the numbers contain rounding errors.

\begin{tabular}{|c|c|c|c|c|c|c|}
\hline$\beta \hbar \omega$ & $n=2^{p+1}$ & $Z_{1 D}^{3}(\beta ; n)$ & $3 \cdot U_{1 D}^{\mathrm{pot}}(\beta ; n)$ & $R_{\text {cut }}$ & $N_{\text {grid }}$ & $t_{\mathrm{CPU}}$ \\
\hline 8.0 & 8 & $9.6578 \times 10^{-6}$ & 0.671429 & 6.0 & 300 & $2.0 \mathrm{~s}$ \\
\hline - & $(8$ & $9.6578 \times 10^{-6}$ & $0.671429)^{*}$ & & & \\
\hline - & 128 & $6.1624 \times 10^{-6}$ & 0.750138 & 6.0 & 300 & $1.0 \mathrm{~s}$ \\
\hline - & 1024 & $6.1506 \times 10^{-6}$ & 0.750498 & 6.0 & 300 & $0.8 \mathrm{~s}$ \\
\hline - & 4096 & $6.1504 \times 10^{-6}$ & 0.750503 & 6.0 & 300 & $0.6 \mathrm{~s}$ \\
\hline - & $(\infty$ & $6.1504 \times 10^{-6}$ & $0.750503) *$ & & & \\
\hline 4.0 & 8 & $2.7908 \times 10^{-3}$ & 0.755902 & 6.0 & 300 & $1.5 \mathrm{~s}$ \\
\hline - & $(8$ & $2.7908 \times 10^{-3}$ & $0.755902)^{*}$ & & & \\
\hline- & 128 & $2.6208 \times 10^{-3}$ & 0.777896 & 6.0 & 300 & $0.9 \mathrm{~s}$ \\
\hline- & 1024 & $2.6201 \times 10^{-3}$ & 0.777985 & 6.0 & 300 & $0.7 \mathrm{~s}$ \\
\hline - & $(\infty$ & $2.6201 \times 10^{-3}$ & $0.777986) *$ & & & \\
\hline 2.0 & 8 & 0.077804 & 0.978570 & 6.0 & 300 & $0.9 \mathrm{~s}$ \\
\hline - & $(8$ & 0.077804 & $0.978570)^{*}$ & & & \\
\hline- & 128 & 0.077018 & 0.984752 & 6.0 & 300 & $0.7 \mathrm{~s}$ \\
\hline- & 1024 & 0.077015 & 0.984776 & 6.0 & 300 & $0.6 \mathrm{~s}$ \\
\hline- & $(\infty$ & 0.077015 & $0.984776) *$ & & & \\
\hline 1.0 & 8 & 0.885268 & 1.620701 & 8.0 & 400 & $1.9 \mathrm{~s}$ \\
\hline- & $(8$ & 0.885268 & $1.620701)^{*}$ & & & \\
\hline- & 128 & 0.883409 & 1.622956 & 8.0 & 400 & $1.3 \mathrm{~s}$ \\
\hline- & 1024 & 0.883402 & 1.622965 & 8.0 & 600 & $3.6 \mathrm{~s}$ \\
\hline- & $(\infty$ & 0.883402 & $1.622965)^{*}$ & & & \\
\hline 0.5 & 8 & 7.75823 & 3.06122 & 10.0 & 500 & $2.8 \mathrm{~s}$ \\
\hline- & $(8$ & 7.75823 & $3.06122)^{*}$ & & & \\
\hline - & 128 & 7.75438 & 3.06224 & 10.0 & 500 & $1.8 \mathrm{~s}$ \\
\hline - & 1024 & 7.75437 & 3.06224 & 10.0 & 1000 & $14.3 \mathrm{~s}$ \\
\hline- & $(\infty$ & 7.75437 & $3.06224)^{*}$ & & & \\
\hline 0.25 & 8 & 63.50981 & 6.03058 & 10.0 & 500 & $2.3 \mathrm{~s}$ \\
\hline- & $(8$ & 63.50981 & $6.03058)^{*}$ & & & \\
\hline - & 128 & 63.50200 & 6.03104 & 10.0 & 500 & $1.4 \mathrm{~s}$ \\
\hline - & 1024 & 63.50195 & 6.03103 & 10.0 & 1500 & $41.3 \mathrm{~s}$ \\
\hline - & $(\infty$ & 63.50221 & $6.03122) *$ & & & \\
\hline
\end{tabular}

tained by integrating $\rho_{\text {ring }}^{\text {(approx })}(\mathbf{r})$ after $(n / 4)$ th iteration; alternatively, Eq. (11) can be integrated with $G^{(n / 2)}(\mathbf{r})$ nonsquared, i.e., when the term for one of the chain ends is only present $(i=1$ or $n-1)$. Naturally, we refer to this ansatz as open ring approximation (ORA).

\section{E. Application of ORA within spherical symmetry}

In this report we apply the suggested open ring approximation in the simplest 3D cases, i.e., with spherically symmetric fields, harmonic and Coulombic ones. Following Allen and Tildesley [32] and Sweatman [19], a considerable simplification of numerical treatment is achieved by use of the Fourier transforms for the two spherically symmetric functions, being parts of the integrand in Eqs. (7) and (15).
That is, on each iteration we first transform the following functions, $H\left(r^{\prime}\right) \equiv G^{(i-1)}\left(r^{\prime}\right) \exp \left[-\beta_{n} \phi\left(r^{\prime}\right)\right]$ and $h\left(\Delta r^{\prime}\right)$ $\equiv \exp \left[-\beta_{n} K_{f}\left(\Delta r^{\prime}\right)^{2}\right]=\exp \left[-\beta_{n} K_{f}\left(\mathbf{r}-\mathbf{r}^{\prime}\right)^{2}\right]$, as

$$
f^{T}(k)=4 \pi \int_{0}^{R_{\text {cut }}} f(r) r^{2} \frac{\sin k r}{k r} d r,
$$

where $f^{T}(k)$ stands for the Fourier transform of $f(r)$. The convolution integrals are, then, taken by merely multiplying the two Fourier transforms with the same values of $k$, and the inverse transform is performed, 

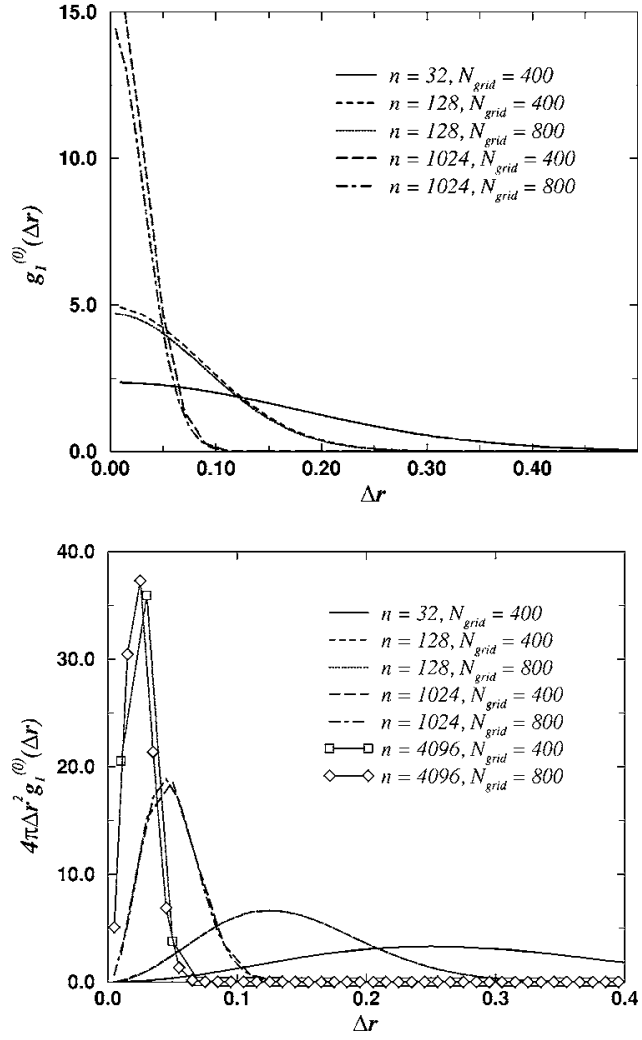

FIG. 1. The normalized harmonic spring distributions, $g_{1}^{(0)}(\Delta r)$ $\sim \exp \left[-\beta_{n} K_{f}(\Delta r)^{2}\right]$ (the same as $h(\Delta r)$ except for normalization), at $\beta^{*}=1$ are presented both in $1 \mathrm{D}$ (top) and 3D (bottom). The effect of increasing the number of monomers/vertices, $n$, is illustrated.

$$
G^{(i)}(r)=\frac{1}{2 \pi^{2}} \int_{0}^{K_{\max }} H^{T}(k) h^{T}(k) k^{2} \frac{\sin k r}{k r} d k .
$$

The integrals, Eq. (12), are evaluated within a finite interval, $0<r<R_{\text {cut }}$, by use of the improved (half-integer) trapezoidal scheme [32]; $R_{\text {cut }}$ is chosen as to cover the most important range of $H\left(r^{\prime}\right)$. The number of Fourier coefficients, $K_{\max }$, is naturally set to the number of bins on the $r$-grid, which presumes the highest possible precision of the transforms. The additional convolutions for the inter-chain interactions [see Eqs. (15) and (19) below] are solved in the same manner.

As above, for the purpose of computational precision, we account for the harmonic normalization factor, $\left(K_{f} \beta / n \pi\right)^{3 / 2}$ $=\left(n / \lambda^{2}\right)^{3 / 2}$ [cf. Eq. (3)], within function $h\left(\Delta r^{\prime}\right)$. Evidently, after $n / 2$ iterations the final calculation of $\rho_{\text {ring }}^{\text {approx }}(r)$ and $Z_{\text {ring }}^{\text {approx }}$, gives the total power of the harmonic norm-factor, $3 n / 2$, which is in accord with Eqs. (1)-(3).

\section{Quantum harmonic oscillator in $3 D$}

The results obtained within ORA for 3D quantum harmonic oscillator are presented in Table II. As expected, for short paths the approximation considerably under-estimates both the energy and the partition function (cf. the rows for $n=8$ in Tables I and II), while the convergence to the known limiting values with increasing number of path vertices, $n>512$, is also observed. At higher inverse temperatures, $\beta^{*}=2, \ldots, 8$, the $n$-convergence is faster, which can be un- derstood as a result of approaching the ground state of $\mathrm{HO}$. Nevertheless, the relative deviations from the exact values $(n \rightarrow \infty)$ are within $1 \%$ for $Z_{3 D}(\beta ; n)$ and $0.05 \%$ for $U_{3 D}^{\mathrm{pot}}(\beta ; n)$.

\section{F. Interparticle interaction: Distinguishable particles}

From Eq. (4) it is clear that the full quantum statistical problem is conveniently split up into simpler problems for a number of distinct permutation classes which can be solved separately. In this report we only address the case of distinguishable particles, i.e., only the identical permutation in Eq. (4) is considered below.

In order to keep the approximation convergence rate, we do not introduce any effective excess free energy term due to additional correlations in a many-particle system, as it is usually done in DFT's for polymer solutions. Instead, we organize a self-consistent iteration for each newly added ( $N$-th) chain-particle interacting with a temporarily fixed distribution of all the rest. Thus, in this case Eq. (7) should include the interaction between vertices with the same indeces on each path, $V_{\text {int }}^{(i)}(|\Delta \mathbf{r}|)$. This is done on the mean-field level by calculating the "correlation integral" for an arbitrary pair of interacting vertices [35],

$$
\tilde{I}_{\text {corr }}\left(\mathbf{r}^{\prime}\right)=\int \frac{\rho_{N}\left(\mathbf{r}^{\prime \prime}\right)}{N} \exp \left[-\beta_{n} V_{\text {int }}\left(\left|\mathbf{r}^{\prime}-\mathbf{r}^{\prime \prime}\right|\right)\right] d \mathbf{r}^{\prime \prime}
$$

which is incorporated in Eq. (7) so that each "monomer" interacts with the mean field generated by its counterparts on the other $(N-1)$ identical paths,

$$
\begin{aligned}
\widetilde{G}_{N}^{(i)}(\mathbf{r})= & \int \widetilde{G}_{N}^{(i-1)}\left(\mathbf{r}^{\prime}\right) \widetilde{I}_{\text {corr }}^{N-1}\left(\mathbf{r}^{\prime}\right) \exp \left[-\beta_{n} \phi\left(\mathbf{r}^{\prime}\right)\right] \\
& \times \exp \left[-\beta_{n} K_{f}\left(\mathbf{r}-\mathbf{r}^{\prime}\right)^{2}\right] d \mathbf{r}^{\prime} .
\end{aligned}
$$

The overall distribution of $N$ distinguishable quantum particles is then obtained as

$$
\widetilde{\rho}_{N}(\mathbf{r}) \sim N n \exp \left[-\beta_{n} \phi(\mathbf{r})\right]\left[\widetilde{G}_{N}^{(n / 2)}(\mathbf{r})\right]^{2} .
$$

Here the tilde sign denotes updated functions, and the iteration is initialized with $\rho_{N}(\mathbf{r})=\rho_{N-1}(\mathbf{r}) N /(N-1), N>1$. As with any self-consistent method, for the purpose of optimization the updated distribution is mixed with the one obtained at the previous stage. The convergence of our calculations appeared, though, to depend rather weakly on the mixing ratio, while the optimum was still achieved by use of the following mixing formula,

$$
\widetilde{\widetilde{\rho}}_{N}(\mathbf{r})=\left[4 \widetilde{\rho}_{N}(\mathbf{r})+\rho_{N}(\mathbf{r})\right] / 5,
$$

which assumes only a few $(<10)$ such iterations per added quantum particle.

One should notice that within this framework all $N$ paths are treated identical, which would also be the case in the corresponding Monte Carlo simulation. However, if it is necessary, one could arrange the iteration so as to keep individual distribution for each particle. Naturally, such details, while increasing the algorithm complexity and the computer 
TABLE II. PI-DFT (ORA) results for the 3D quantum harmonic oscillator obtained within the open ring approximation. The notation is analogous to that of Table I with the only difference: the range of numerical integration for the Fourier transforms is $\left(0, R_{\text {cut }}\right)$, whereas $3 \mathrm{D}$ integrals are solved by the inverse convolution transform.

\begin{tabular}{|c|c|c|c|c|c|c|}
\hline$\beta \hbar \omega$ & $n$ & $Z_{3 D}(\beta ; n)$ & $U_{3 D}^{\mathrm{pot}}(\beta ; n)$ & $R_{\text {cut }}$ & $N_{\text {grid }}$ & $t_{\mathrm{CPU}}$ \\
\hline 8.0 & 8 & $7.513 \times 10^{-7}$ & 0.67105 & 5.12 & 512 & $0.3 \mathrm{~s}$ \\
\hline- & 128 & $5.116 \times 10^{-6}$ & 0.75011 & 5.12 & 512 & $3.6 \mathrm{~s}$ \\
\hline- & 1024 & $6.008 \times 10^{-6}$ & 0.75049 & 5.12 & 512 & $28 \mathrm{~s}$ \\
\hline- & 4096 & $6.114 \times 10^{-6}$ & 0.75050 & 5.12 & 512 & $2 \mathrm{~m}$ \\
\hline - & $(\infty$ & $6.150 \times 10^{-6}$ & $0.75050)^{*}$ & & & \\
\hline 4.0 & 8 & $6.6760 \times 10^{-4}$ & 0.74473 & 5.12 & 512 & $0.3 \mathrm{~s}$ \\
\hline - & 128 & $2.3791 \times 10^{-3}$ & 0.77702 & 5.12 & 512 & $3.6 \mathrm{~s}$ \\
\hline - & 1024 & $2.5885 \times 10^{-3}$ & 0.77787 & 5.12 & 512 & $28 \mathrm{~s}$ \\
\hline - & 4096 & $2.6122 \times 10^{-3}$ & 0.77796 & 5.12 & 768 & $4 \mathrm{~m}$ \\
\hline- & $(\infty$ & $2.6201 \times 10^{-3}$ & $0.77799) *$ & & & \\
\hline 2.0 & 8 & 0.031484 & 0.92072 & 7.68 & 512 & $0.3 \mathrm{~s}$ \\
\hline- & 128 & 0.072461 & 0.98057 & 7.68 & 512 & $3.6 \mathrm{~s}$ \\
\hline- & 1024 & 0.076442 & 0.98426 & 7.68 & 512 & $28 \mathrm{~s}$ \\
\hline- & 4096 & 0.076987 & 0.98464 & 7.68 & 768 & $4 \mathrm{~m}$ \\
\hline- & $(\infty$ & 0.077015 & $0.98478) *$ & & & \\
\hline 1.0 & 8 & 0.4256 & 1.4699 & 10.24 & 512 & $0.3 \mathrm{~s}$ \\
\hline- & 128 & 0.8400 & 1.6123 & 10.24 & 512 & $3.6 \mathrm{~s}$ \\
\hline- & 1024 & 0.8778 & 1.6216 & 10.24 & 512 & $28 \mathrm{~s}$ \\
\hline- & 4096 & 0.8820 & 1.6226 & 10.24 & 1024 & $8 \mathrm{~m}$ \\
\hline- & $(\infty$ & 0.8834 & $1.62297)^{*}$ & & & \\
\hline 0.5 & 8 & 3.9091 & 2.7357 & 10.24 & 512 & $0.3 \mathrm{~s}$ \\
\hline- & 128 & 7.3947 & 3.0395 & 10.24 & 512 & $3.6 \mathrm{~s}$ \\
\hline- & 1024 & 7.7081 & 3.0594 & 10.24 & 512 & $28 \mathrm{~s}$ \\
\hline- & 4096 & 7.7428 & 3.0615 & 10.24 & 1024 & $8 \mathrm{~m}$ \\
\hline - & $(\infty$ & 7.7544 & $3.0622)^{*}$ & & & \\
\hline 0.25 & 8 & 32.327 & 5.3680 & 10.24 & 512 & $0.3 \mathrm{~s}$ \\
\hline- & 128 & 60.127 & 5.9846 & 10.24 & 512 & $3.6 \mathrm{~s}$ \\
\hline - & 1024 & 62.586 & 6.0250 & 10.24 & 768 & $1 \mathrm{~m}$ \\
\hline - & 4096 & 62.858 & 6.0294 & 10.24 & 1536 & $16 \mathrm{~m}$ \\
\hline- & $(\infty$ & 63.502 & $6.0312)^{*}$ & & & \\
\hline
\end{tabular}

demand, do not bring any additional information in most physically relevant cases. But this way it is possible to see, for instance, that a proton, $\mathrm{H}^{+}$, can attract (bound) only one electron in the hydrogen atom. That is, the converged distribution for the second electron is considerably shifted to the periphery of the integration cell, while the distribution of the first one is affected very slightly, see Fig. 2.

\section{G. Energy evaluation}

Above we described the general numerical methods yielding the monomer density distribution and the corresponding partition function for ideal chains/paths in the presence of external field(s). Thermodynamic quantities, such as potential and kinetic contributions to the total energy in the system, can be obtained by averaging of the appropriate estimators, being functions of the particle coordinates. In particular, the average potential energy of a quantum particle in the external field, $\phi(\mathbf{r})$, is calculated simply as

$$
U_{1}^{\mathrm{pot}}(\beta)=\int \phi(\mathbf{r}) \rho\left(\mathbf{r} ; \beta_{n}\right) d \mathbf{r},
$$

with $\rho\left(\mathbf{r} ; \beta_{n}\right)$ properly normalized beforehand. It is clear that with $N$ noninteracting particles, $U_{N}^{\text {pot }}(\beta)=N U_{1}^{\text {pot }}(\beta)$, which can also be seen as a result of renormalization of $\rho\left(\mathbf{r} ; \beta_{n}\right)$ by the factor of $N$. If several distinguishable particles do interact with each other, besides $U_{N}^{\text {pot }}(\beta)$, one has to account for the average contribution of the interparticle interaction, 

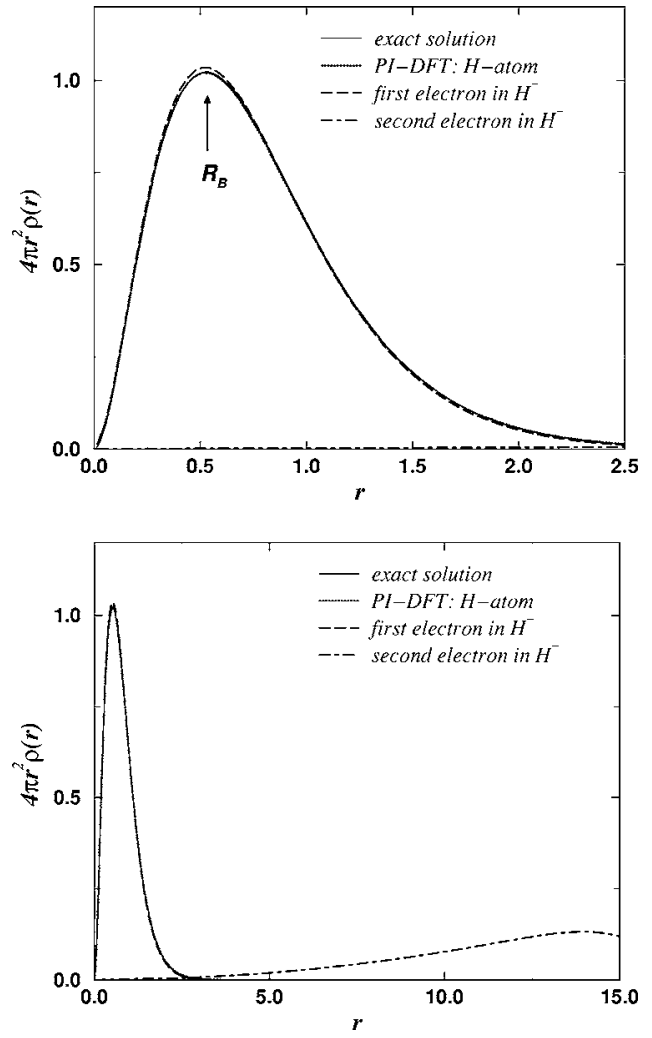

FIG. 2. The electron distributions in the hydrogen atom and in the artificial $\mathrm{H}^{-}$system (proton and two electrons). In both cases $n=2048$ and $T=10000 \mathrm{~K}\left(R_{\text {cut }}=20.48 \AA, N_{\text {grid }}=1024\right)$. The known exact solution of the Schrödinger equation is also shown for comparison, and the first Bohr radius, $R_{B}=0.529 \AA$, is marked by the arrow.

$$
\begin{aligned}
U_{N}^{\text {int }}(\beta)= & \frac{N(N-1)}{2} \int \frac{\rho_{N}\left(\mathbf{r} ; \beta_{n}\right)}{N} d \mathbf{r} \int \frac{\rho_{N}\left(\mathbf{r}^{\prime} ; \beta_{n}\right)}{N} \\
& \times \exp \left[-\beta_{n} V_{\text {int }}\left(\left|\mathbf{r}-\mathbf{r}^{\prime}\right|\right)\right] V_{\text {int }}\left(\left|\mathbf{r}-\mathbf{r}^{\prime}\right|\right) d \mathbf{r}^{\prime},
\end{aligned}
$$

where the integral is calculated for a pair of particles and the result is multiplied by the number of pairs present. In order to obtain the kinetic energy, it is common to introduce a so-called virial estimator $[25,26]$ which, based on the virial theorem, gives in the case of Coulombic interactions,

$$
U_{N}^{\mathrm{kin}}=-\frac{1}{2}\left(U_{N}^{\mathrm{pot}}+U_{N}^{\mathrm{int}}\right)
$$

Thus, the total energy in a quantum system of $N$ distinguishable particles is estimated as

$$
U_{N}^{\mathrm{tot}}=U_{N}^{\mathrm{pot}}+U_{N}^{\mathrm{int}}+U_{N}^{\mathrm{kin}}=\frac{1}{2}\left(U_{N}^{\mathrm{pot}}+U_{N}^{\mathrm{int}}\right) .
$$

\section{RESULTS}

As is well-known, the electrostatic field in the vicinity of a charge, such as $\mathrm{H}^{+}$, is so strong that the PI-treatment of the electron-nucleus interaction requires paths with vertices very close to each other, which implies a rather high effective temperature, $T_{n}=n T$. That is, in order to obtain plausible results, one has to use sufficiently large $n$. While this fact is the known "bottle-neck" with Monte Carlo simulations, it is consistent with the limiting behaviour of ORA, giving much less trouble with PI-DFT. In all our calculations below we use $n>1000$, and most of them take only several minutes to execute.

Yet, because of $1 / r$ dependence of the Coulombic potential, in the close proximity of a nucleus the numerical integration with finite $n$ is a subttle business. Therefore, we adopt a technique of smoothing the potential which is commonly used in PIMC simulations [26]. In particular, we employ the parabolic smoothing at short distances,

$$
\phi_{s}(r)=a r^{2}+b, \quad r<R_{s},
$$

where the parameters are chosen so as to keep the continuity of the first derivative of the smoothed potential at $r=R_{s}$, namely: $a=-e^{2} /\left(2 R_{s}^{3}\right), b=3 e^{2} /\left(2 R_{s}\right)$. Notice, unlike with the PIMC method where $R_{s}$ has to be set to about $0.1 \AA$, in our PI-DFT calculations we could reduce $R_{s}$ as low as $0.005 \ldots 0.04 \AA$, depending on $n$ and $T$ used. Thus, smoothing was applied only in a few (1-5) bins of integration, closest to the origin, which reveals a considerable gain in precision as compared to PIMC. Moreover, with sufficiently long path chains the smoothing parameter was set to $0.001 \AA$, which implies, in fact, no smoothing at all. Numerical integration is still possible without encountering the infinity issue at the origin, because within the half-integer trapezoidal scheme we only calculate the Coulombic potential in the center of each bin, and a linear extrapolation to zero is implicitly applied in the very first integration bin.

\section{A. Hydrogen atom}

For obtaining a reasonable estimate of the ground state energy in $\mathrm{H}$-atom, it was sufficient to use $n=2048$ at $T=10000 \mathrm{~K}$, or $n=4096$ at $T=8000 \mathrm{~K}$. As one can see in Fig. 2, the calculated distribution practically coincides with the exact one, although there are important deviations from the latter on the smaller scale which are discussed below. In the same figure we presented two separate distributions obtained in the case of two distiguishable electrons interacting with each other in the field of $\mathrm{H}^{+}$, thus, making up the (artificial) $\mathrm{H}^{-}$system. The obvious interpretation is, when two electrons of opposite spin are in the neighborhood of the proton, one of them is bounded within the H-atom, whereas the other escapes.

In Table III we give an extended presentation of data obtained for the ground state of $\mathrm{H}$-atom with a variety of parameters. It should be stressed that even though all the energy estimates do not deviate from its known value, $E_{g}(H)$ $=-13.606 \mathrm{eV}$, more than $2 \%$, at this stage it is important to learn the convergence of calculations with the two crucial parameters, $n$ and $R_{s}$. For example, $n=1024$ is not large enough even at $T=10000 \mathrm{~K}$ and $R_{s}=0.03 \AA$. Actually, the energy estimate appears to be good merely due to cancellation of errors in the electron distribution: at short distances, $r<0.3 \AA, \rho(r)$ is about $1-3 \%$ overestimated (in spite of 
TABLE III. PI-DFT (ORA) results for the ground (bound) electron state in H-atom. The notation is similar to that in Table II. Notice that these results were obtained with the fast Fourier transform method which reduced the $N_{\text {grid }}$-dependence of CPU time to $O\left(N_{\text {grid }} l n N_{\text {grid }}\right)$ instead of $O\left(N_{\text {grid }}^{2}\right)$ (cf. Table II). The energy is to be compared to the exact value, $E_{g}(H)=-13.606 \mathrm{eV}$.

\begin{tabular}{|c|c|c|c|c|c|c|}
\hline$T(\mathrm{~K})$ & $n$ & $U^{\mathrm{tot}}(\mathrm{eV})$ & $R_{s}(\AA)$ & $R_{\text {cut }}(\AA)$ & $N_{\text {grid }}$ & $t_{\mathrm{CPU}}(\mathrm{FFT})$ \\
\hline 10000 & 1024 & -13.546 & 0.04 & 10.24 & 512 & $2.3 \mathrm{~s}$ \\
\hline- & 1024 & -13.605 & 0.03 & 10.24 & 512 & $2.3 \mathrm{~s}$ \\
\hline- & 1024 & -13.665 & 0.02 & 10.24 & 512 & $2.3 \mathrm{~s}$ \\
\hline- & 2048 & -13.523 & 0.03 & 10.24 & 768 & $7.0 \mathrm{~s}$ \\
\hline- & 2048 & -13.562 & 0.02 & 10.24 & 768 & $7.0 \mathrm{~s}$ \\
\hline- & 2048 & -13.606 & 0.01 & 10.24 & 768 & $7.0 \mathrm{~s}$ \\
\hline- & 4096 & -13.530 & 0.02 & 10.24 & 1024 & $19.0 \mathrm{~s}$ \\
\hline- & 4096 & -13.552 & 0.01 & 10.24 & 1024 & $19.0 \mathrm{~s}$ \\
\hline- & 4096 & -13.570 & 0.005 & 10.24 & 1024 & $19.0 \mathrm{~s}$ \\
\hline- & 8192 & -13.551 & 0.001 & 10.24 & 1024 & $38.0 \mathrm{~s}$ \\
\hline- & 8192 & -13.550 & 0.001 & 10.24 & 2048 & $76.0 \mathrm{~s}$ \\
\hline- & 8192 & -13.548 & 0.001 & 15.36 & 2048 & $76.0 \mathrm{~s}$ \\
\hline- & 8192 & -13.544 & 0.001 & 20.48 & 4096 & $2.5 \mathrm{~m}$ \\
\hline- & 16384 & -13.537 & 0.001 & 20.48 & 4096 & $5.0 \mathrm{~m}$ \\
\hline 8000 & 1024 & -13.572 & 0.05 & 10.24 & 768 & $3.5 \mathrm{~s}$ \\
\hline- & 1024 & -13.638 & 0.04 & 10.24 & 768 & $3.5 \mathrm{~s}$ \\
\hline- & 2048 & -13.591 & 0.03 & 10.24 & 768 & $7.0 \mathrm{~s}$ \\
\hline- & 2048 & -13.633 & 0.02 & 10.24 & 1024 & $9.4 \mathrm{~s}$ \\
\hline- & 4096 & -13.588 & 0.02 & 10.24 & 1024 & $19.0 \mathrm{~s}$ \\
\hline- & 4096 & -13.613 & 0.01 & 10.24 & 1024 & $19.0 \mathrm{~s}$ \\
\hline - & 8192 & -13.593 & 0.01 & 10.24 & 1024 & $38.0 \mathrm{~s}$ \\
\hline- & 8192 & -13.603 & 0.005 & 15.36 & 2048 & $76.0 \mathrm{~s}$ \\
\hline - & 16384 & -13.595 & 0.005 & 10.24 & 2048 & $2.5 \mathrm{~m}$ \\
\hline - & 16384 & -13.597 & 0.001 & 15.36 & 2048 & $2.5 \mathrm{~m}$ \\
\hline - & 25000 & -13.593 & 0.001 & 20.48 & 4096 & $7.6 \mathrm{~m}$ \\
\hline 4000 & 16384 & -13.616 & 0.005 & 10.24 & 2048 & $2.5 \mathrm{~m}$ \\
\hline - & 32000 & -13.612 & 0.001 & 10.24 & 4096 & $10.0 \mathrm{~m}$ \\
\hline - & 48000 & -13.609 & 0.001 & 10.24 & 4096 & $15.0 \mathrm{~m}$ \\
\hline- & 96000 & -13.607 & 0.001 & 10.24 & 4096 & $30.0 \mathrm{~m}$ \\
\hline
\end{tabular}

smoothing), same at $r>0.7 \AA$ (because $T$ is too high), while in the intermediate interval it is correspondingly underestimated. This observation is also true for $n=2048$, although the deviations are smaller in this case.

Furthermore, as one can see from the energy values obtained with larger $n, T=10000 \mathrm{~K}$ is slightly "too high" temperature, i.e., the observed quantum state is not really the pure ground state of $\mathrm{H}$-atom, hence a slight increase in the converged energy estimates, with $n>4096$ and without smoothing $\left(R_{S}=0.001 \AA\right)$. A more attentive investigation of the electron distribution on small scale reveals that at $T$ $>8000 \mathrm{~K}$ (approximately) its long-range "tail" does not vanish but, in opposite, at $r>8 \AA$ it starts to slowly accumulate, which is observed with any $n$ and $R_{\text {cut }}>9 \AA$. This fact might be easily overlooked in the corresponding PIMC simulations, because the actual long-range probabilities are at most of the order of $10^{-4}$. The situation is, however, different at lower temperatures, $T<8000 \mathrm{~K}$, in which case the probability of finding the electron at distances $r>3 R_{B}$ is quickly diminishing, as expected for the bound electron state in
$\mathrm{H}$-atom. To resume, the data in Table III do converge to the ground state of $\mathrm{H}$-atom with decreasing $T$, and at sufficiently large $n$ the smoothing procedure can be skipped.

\section{B. Helium ion and atom, lithium ions}

The ions $\mathrm{He}^{+}$and $\mathrm{Li}^{2+}$ are different from $\mathrm{H}$-atom only by virtue of the charge of their nuclei. So, by setting the latter to 2 or 3 and executing the PI-DFT calculation at sufficiently low temperatures, $T<30000 \mathrm{~K}$, one obtains the data that converge to the ground states of the electron subsystems in $\mathrm{He}^{+}$and $\mathrm{Li}^{2+}$; see Table IV. In the cases of He-atom and $\mathrm{Li}^{+}$, where two electrons are present, one also has to account for the electron-electron correlations. In the ground state the two electrons have opposite spins and occupy the same quantum state. Therefore they can be treated as two distinguishable particles, for which only the identical permutation remains in Eq. (4), whereas the electron-electron correlations/ interactions are included within the self-consistent procedure, Eqs. (15) and (19). After 6 iterations the relative dif- 
TABLE IV. PI-DFT (ORA) results for the ground electronic states in helium and lithium. The notation is the same as in Table III. The values of $U^{\text {tot }}$ are to be compared to the known data, $E_{g}\left(\mathrm{He}^{+}\right)=-54.4 \mathrm{eV}, E_{g}(\mathrm{He})=-79.1 \mathrm{eV}, E_{g}\left(\mathrm{Li}^{2+}\right)=-121.8 \mathrm{eV}, E_{g}\left(\mathrm{Li}^{+}\right)=-197.4 \mathrm{eV}$.

\begin{tabular}{|c|c|c|c|c|c|c|c|}
\hline & $T(\mathrm{~K})$ & $n$ & $U^{\text {tot }}(\mathrm{eV})$ & $R_{s}(\AA)$ & $R_{\text {cut }}(\AA)$ & $N_{\text {grid }}$ & $t_{\mathrm{CPU}}(\mathrm{FFT})$ \\
\hline $\mathrm{He}^{+}$ & 20000 & 4096 & -54.516 & 0.01 & 10.24 & 1024 & $19.0 \mathrm{~s}$ \\
\hline $\mathrm{He}$ & 20000 & 4096 & -77.777 & 0.01 & 10.24 & 1024 & $2.0 \mathrm{~m}$ \\
\hline $\mathrm{He}^{+}$ & 20000 & 4096 & -54.718 & 0.005 & 15.36 & 2048 & $38.0 \mathrm{~s}$ \\
\hline $\mathrm{He}$ & 20000 & 4096 & -78.038 & 0.005 & 15.36 & 2048 & $4.0 \mathrm{~m}$ \\
\hline $\mathrm{He}^{+}$ & 20000 & 8192 & -54.499 & 0.001 & 10.24 & 2048 & $76.0 \mathrm{~s}$ \\
\hline $\mathrm{He}$ & 20000 & 8192 & -77.817 & 0.001 & 10.24 & 2048 & $8.0 \mathrm{~m}$ \\
\hline $\mathrm{He}^{+}$ & 20000 & 8192 & -54.550 & 0.001 & 20.48 & 4096 & $2.5 \mathrm{~m}$ \\
\hline $\mathrm{He}$ & 20000 & 8192 & -77.760 & 0.001 & 20.48 & 4096 & $16.0 \mathrm{~m}$ \\
\hline $\mathrm{He}^{+}$ & 20000 & 16384 & -54.473 & 0.001 & 20.48 & 4096 & $5.0 \mathrm{~m}$ \\
\hline $\mathrm{He}$ & 20000 & 16384 & -77.667 & 0.001 & 20.48 & 4096 & $32.0 \mathrm{~m}$ \\
\hline $\mathrm{He}^{+}$ & 12000 & 8192 & -54.707 & 0.001 & 20.48 & 4096 & $2.5 \mathrm{~m}$ \\
\hline $\mathrm{He}$ & 12000 & 8192 & -78.289 & 0.001 & 20.48 & 4096 & $16.0 \mathrm{~m}$ \\
\hline $\mathrm{He}^{+}$ & 8000 & 16384 & -54.608 & 0.001 & 15.36 & 4096 & $5.0 \mathrm{~m}$ \\
\hline $\mathrm{He}$ & 8000 & 16384 & -78.150 & 0.001 & 15.36 & 4096 & $32.0 \mathrm{~m}$ \\
\hline $\mathrm{Li}^{2+}$ & 32000 & 8192 & -122.600 & 0.005 & 20.48 & 4096 & $2.5 \mathrm{~m}$ \\
\hline $\mathrm{Li}^{+}$ & 32000 & 8192 & -197.135 & 0.005 & 20.48 & 4096 & $16.0 \mathrm{~m}$ \\
\hline $\mathrm{Li}^{2+}$ & 32000 & 16384 & -122.651 & 0.001 & 20.48 & 4096 & $5.0 \mathrm{~m}$ \\
\hline $\mathrm{Li}^{+}$ & 32000 & 16384 & -197.219 & 0.001 & 20.48 & 4096 & $32.0 \mathrm{~m}$ \\
\hline $\mathrm{Li}^{2+}$ & 24000 & 16384 & -122.716 & 0.001 & 15.36 & 4096 & $5.0 \mathrm{~m}$ \\
\hline $\mathrm{Li}^{+}$ & 24000 & 16384 & -197.328 & 0.001 & 15.36 & 4096 & $32.0 \mathrm{~m}$ \\
\hline $\mathrm{Li}^{2+}$ & 16000 & 24000 & -122.725 & 0.001 & 15.36 & 4096 & $7.5 \mathrm{~m}$ \\
\hline $\mathrm{Li}^{+}$ & 16000 & 24000 & -197.342 & 0.001 & 15.36 & 4096 & $45.0 \mathrm{~m}$ \\
\hline
\end{tabular}

ference between the final energy estimate and the value obtained at the previous stage is already within $0.005 \%$.

As is seen from the data in Table IV, the convergence to the ground states is achieved by reducing the temperature and increasing the number of vertices, $n$. Again, like with $\mathrm{H}$-atom, by carefully choosing the smoothing distance, $R_{s}$, one can obtain reasonable estimates for the energies with relatively small $n=4096$ at $T=20000 \mathrm{~K}$ for $\mathrm{He}$, and $n$ $=8192$ at $T=32000 \mathrm{~K}$ for $\mathrm{Li}$. The latter fact is, of course, attributed to compensation of systematic errors in the electron distribution which is overestimated at short and long distances and underestimated around its maximum as discussed above. There is also a certain inconsistency in convergence of the results obtained with one and two electrons at the same $T$. For instance, any $T<20000 \mathrm{~K}$ appears to be sufficiently low for obtaining the converged, i.e., vanishing at long distances, electron distribution in the case of $\mathrm{He}^{+}$, whereas the distribution of two interacting electrons in $\mathrm{He}$ atom still has a secondary minimum at $r>4.5 \AA$ unless the temperature is lowered down to about $8000 \mathrm{~K}$. Accordingly, the convergence of energy estimates for the two electron system (He atom) is much slower than in the case of $\mathrm{He}^{+}$. Thus, the overall observation is rather natural: a single electron in the field of two protons is bounded much stronger than any of the paired electrons in the same field. The same trend is observed in the electron subsystem of lithium as well.

\section{CONCLUSION}

The presented path integral density functional approach has shown itself quite well in calculation of the quantum particle distributions and energies in a number of quantum systems, including 1D and 3D quantum harmonic oscillators and simple atomic systems. The two suggested integration schemes provide path integral statistics orders of magnitude more accurate than the corresponding Monte Carlo simulation. This feature makes it possible to calculate not only ordinary statistical averages but also the partition functions of the classical chains being isomorphic counterparts of quantum particles. In fact, the numerically exact solution that is tractable in 1D (and likely in 2D) is straightforward to apply to low-dimensional quantum dots and it might also be valuable for studying Bose-Einstein condensation in traps or superfluid behavior of liquids.

Despite the fact that the open ring approximation converges only in the limit of long chains, as soon as the problem can be re-formulated in terms of convolution integrals (quickly solved by use of fast Fourier transforms) the numerical integration is very fast, and thousands of vertices can be treated in some seconds or minutes. From this point of view, it would be interesting to see how the method performs for problems in actual two and three dimensions, without using the benefits of spherical symmetry. While in this work we applied PI-DFT in the case of distinguishable particles, it 
should also be possible to develop the method further and to attack the sign (exchange) problem which still remains the major obstacle in application of the general path integral formalism, especially with the Coulombic potential. We address this issue in our next paper.

\section{ACKNOWLEDGEMENT}

A.B. expresses his gratitude to Dr. Jan Forsman (Theoretical Chemistry, Lund University) for helpful discussions clarifying the ideas behind polymer DFT's, on the early stage of this work.
[1] P. Hohenberg and W. Kohn, Phys. Rev. B 136, 864 (1964).

[2] W. Kohn and L. J. Sham, Phys. Rev. A 140, 1133 (1965).

[3] N. D. Mermin, Phys. Rev. A 137, 1441 (1965).

[4] J. L. Lebowitz and J. K. Percus, J. Math. Phys. 4, 116, (1963).

[5] B. M. Ladanyi and D. Chandler, J. Chem. Phys. 62, 4308 (1975).

[6] D. Chandler and L. R. Pratt, J. Chem. Phys. 65, 2925 (1976).

[7] D. Chandler and P. G. Wolynes, J. Chem. Phys. 74, 4078 (1981).

[8] D. Chandler, J. D. McCoy, and S. J. Singer, J. Chem. Phys. 85, 5971, (1986).

[9] J. D. McCoy, S. W. Rick, and A. D. J. Haymet, J. Chem. Phys. 90, 4622 (1989).

[10] J. D. McCoy, S. W. Rick, and A. D. J. Haymet, J. Chem. Phys. 92, 3034 (1990).

[11] S. W. Rick, J. D. McCoy, and A. D. J. Haymet, J. Chem. Phys. 92, 3040 (1990)

[12] C. E. Woodward, J. Chem. Phys. 94, 3183 (1991).

[13] C. E. Woodward, J. Chem. Phys. 97, 695 (1992).

[14] A. Yethiraj and C. E. Woodward, J. Chem. Phys. 102, 5499 (1995).

[15] C. N. Patra and A. Yethiraj, J. Chem. Phys. 112, 1579 (2000).

[16] Y.-X. Yu and J. Wu, J. Chem. Phys. 116, 7094, (2002); 117, 2368 (2002).

[17] C. Gu, G.-H. Gao, and Y.-X. Yu, J. Chem. Phys. 119, 488 (2003).

[18] H. Löwen, J. Phys.: Condens. Matter 14, 11897 (2002).

[19] M. B. Sweatman, J. Phys.: Condens. Matter 15, 3875 (2003).

[20] J. Forsman, C. E. Woodward, and B. C. Freasier, J. Chem. Phys. 117, 1915 (2002).

[21] J. Forsman, A. Broukhno, B. Jönsson, and T. A. Kesson, J. Chem. Phys. 120, 413 (2003).
[22] R. P. Feynman and A. R. Hibbs, Quantum Mechanics and Path Integrals (McGraw-Hill, New York, 1965).

[23] R. P. Feynman, Statistical Mechanics (McGraw-Hill, New York, 1972).

[24] C. Chakravarty, J. Chem. Phys. 99, 8038 (1993).

[25] A. P. Lyubartsev and P. N. Vorontsov-Velyaminov, Phys. Rev. A 48, 4075 (1993).

[26] P. N. Vorontsov-Velyaminov, M. O. Nesvit, and R. I. Gorbunov, Phys. Rev. E 55, 1979 (1997).

[27] P. N. Vorontsov-Velyaminov and A. P. Lyubartsev, J. Phys. A 36, 685 (2003).

[28] P. N. Vorontsov-Velyaminov, D. A. Ivanov, S. D. Ivanov, and A. V. Broukhno, Colloids Surf., A 148, 171 (1999).

[29] S. L. Mielke and D. G. Truhlar, J. Chem. Phys. 114, 621 (2001).

[30] C. Chakravarty, M. C. Gordillo, and D. M. Ceperley, J. Chem. Phys. 109, 2123 (1998).

[31] C. Chakravarty, J. Chem. Phys. 116, 8938 (2002).

[32] M. P. Allen and D. J. Tildesley, Computer Simulation of Liquids (Oxford University Press, Oxford, 1989).

[33] In fact, we made an attempt to use the weighted density approximation developed for rings by Sweatman [19], and our result for the ground state of hydrogen atom was unsatisfactory (not reported).

[34] We assume that the Hamiltonian does not depend on the spin variable.

[35] $I_{\text {corr }}(\mathbf{r})$ measures the probability of finding a sample charge in the mean field generated by a representative vertex on another chain. Since all the vertices within a chain are, on average, distributed identically, $I_{\text {corr }}(\mathbf{r})$ is the same for any pair of interacting vertices. 